\title{
On the Weinstein conjecture in higher dimensions
}

Peter Albers and Helmut Hofer*

\begin{abstract}
The existence of a "Plastikstufe" for a contact structure implies the Weinstein conjecture for all supporting contact forms.
\end{abstract}

Mathematics Subject Classification (2000). 53D10, 53D35, 37J45.

Keywords. Contact structure, Reeb vector field, over-twisted, Plastikstufe, Weinstein conjecture.

\section{Introduction and main result}

A one-form $\lambda$ on an odd-dimensional manifold $M^{2 n-1}$ is called a contact form, provided $\lambda \wedge d \lambda^{n-1}$ is a volume-form. Associated to a contact form $\lambda$ we have the Reeb vector field $X$ defined by

$$
i_{X} \lambda=1 \text { and } i_{X} d \lambda=0
$$

and the contact structure $\xi=\operatorname{ker}(\lambda)$. In 1978, A. Weinstein, [21], motivated by a result of P. Rabinowitz, [16], and one of his own results, [20], made the following conjecture:

A Reeb vector field on a closed manifold $M^{2 n-1}$ admits a periodic orbit.

The first break-through on this conjecture was obtained by C. Viterbo, [19], showing that compact energy surfaces in $\mathbb{R}^{2 n}$ of contact-type have periodic orbits. Extending Gromov's theory of pseudoholomorphic curves, [3], to symplectized contact manifolds, H. Hofer, [4], related the Weinstein conjecture to the existence of certain pseudoholomorphic curves. He showed that in dimension three the Weinstein conjectures holds in many cases. In particular, he showed that Reeb vector fields associated to over-twisted contact structures admit periodic orbits. Recently the Weinstein conjecture in dimension three was completely settled by C. Taubes, [17, 18], who exploited relationships between Seiberg-Witten-Floer homology, [12], and embedded contact

* Research partially supported by NSF grant DMS-0603957 and DFG grant AL 904/1-1. 
homology, [11], in order to construct holomorphic curves in the symplectized contact manifold out of nontrivial Seiberg-Witten-Floer homology classes. For more references on the Weinstein conjecture see [6].

In this note we show that many Reeb vector fields on higher dimensional closed manifolds have periodic orbits generalizing the main result from [4]. Our existence result is closely connected to the interesting attempt by K. Niederkrüger [13] to generalize the three-dimensional notion of an overtwisted contact structure. He introduced the concept of a Plastikstufe which currently seems to be the most compelling generalisation given recent further developments by F. Presas, [15], and K. Niederkrüger and O. van Koert, [14].

Let us denote by $(M, \xi)$ a pair consisting of a closed manifold $M$ of dimension $2 n-1$ and a co-oriented contact structure $\xi$. We denote by $\mathbb{D}^{2}$ the closed unit disk in $\mathbb{C}$ with coordinates $x+i y$.

Definition 1.1. We say that $(M, \xi)$ contains a Plastikstufe with singular set $S$ provided $M$ admits a closed submanifold $S$ of dimension $n-2$ and an embedding $\iota: \mathbb{D}^{2} \times S \rightarrow M$ with $\iota(\{0\} \times S)=S$ having the following properties:

(1) There exists a contact form $\lambda_{\mathrm{PS}}$ inducing $\xi$ so that the one-form $\beta:=\iota^{*} \lambda_{\mathrm{PS}}$ satisfies $\beta \wedge d \beta=0$ and moreover $\beta \neq 0$ on $\left(\mathbb{D}^{2} \backslash\{0\}\right) \times S$. Near $\{0\} \times S$ the form $\beta$ is given by $\beta=x d y-y d x$ and the pull-back of $\beta$ to $\partial \mathbb{D}^{2} \times S$ vanishes.

(2) The complement of $\{0\} \times S$ in $\left(\mathbb{D}^{2} \backslash \partial \mathbb{D}^{2}\right) \times S$ is smoothly foliated by $\beta$ via an $S^{1}$-family of leaves diffeomorphic to $(0,1) \times S$, where one of the ends converges to the singular set $\{0\} \times S$ and the other is asymptotic to the leaf $\partial \mathbb{D}^{2} \times S$.

The set $\mathcal{P S}(S)=\iota\left(\mathbb{D}^{2} \times S\right)$ is called the Plastikstufe.

Let us observe that the existence of a Plastikstufe for a given contact structure involves the existence of a certain inducing contact form. This is different from the three-dimensional case where an over-twisted disk is defined only in terms of the contact structure and does not require the existence of a particular contact form. In the following we shall call a closed co-oriented contact manifold $(M, \xi)$ PS-overtwisted provided there exists a contact form $\lambda_{\mathrm{PS}}$ inducing $\xi$ containing a Plastikstufe. Recently Niederkrüger and van Koert showed that every odd-dimensional sphere $S^{2 n-1}$ with $n \geq 3$ has a contact structure admitting a Plastikstufe. If now $\left(M^{2 n-1}, \xi\right)$ is a cooriented contact manifold then a connected sum with a PS-overtwisted sphere admits by standard arguments a contact structure which is PS-overtwisted. In particular, any closed manifold of dimension $2 n-1$ admitting a co-oriented contact structure also admits a PS-overtwisted contact structure. Our main result is the following theorem.

Theorem. Let $(M, \xi)$ be a closed PS-overtwisted contact manifold. Then every Reeb vector field associated to a contact form $\lambda$ inducing $\xi$ has a contractible periodic orbit. 
Remark 1.2. In [13] Niederkrüger shows that a PS-overtwisted contact structure does not have a semi-positive symplectic filling. We noticed that some of his idea combined with ideas from [4] lead to the above theorem. We also observed that the limitation to semi-positive fillings is not necessary and can be removed using polyfolds [5]. This will be discussed in a forthcoming paper.

\section{Background}

All material in this section is taken from [13].

2.1. Local normal form. Let $(M, \lambda)$ contain a Plastikstufe $\mathcal{P S}(S)$. In $[13$, Section 3.1] it is proved that there exist constants $\varepsilon, C>0$ and an open set $V$ in the symplectic manifold $\left((-\varepsilon, 0] \times M, d\left(e^{s} \lambda\right)\right)$ such that $\{0\} \times S \subset V$ and $V$ is symplectomorphic to the subset

$$
U:=\left\{\begin{array}{l|l}
\left(\left(z_{1}, z_{2}\right),(q, p)\right) \in \mathbb{C}^{2} \times T^{*} S \mid \begin{array}{l}
-C<\operatorname{Re}\left(z_{1}\right) \leq 0 \\
-C<\operatorname{Im}\left(z_{1}\right)<C \\
\operatorname{Re}\left(z_{1}\right)+\frac{1}{4}\left|z_{2}\right|^{2}+\frac{1}{2}\|p\|^{2} \leq 0
\end{array}
\end{array}\right\}
$$

of $\mathbb{C}^{2} \times T^{*} S$ which carries its natural symplectic structure. Moreover, $M \cap V$ corresponds to equality in the last equation and $\mathcal{P S}(S) \cap V$ to equality and $\operatorname{Im}\left(z_{1}\right)=0, p=0$.

2.2. Bishop family. The local model $U$ contains a natural $(n-1)$-dimensional Bishop family given by

$$
\begin{aligned}
u_{t_{0}, q_{0}}: \mathbb{D}^{2} & \longrightarrow \mathbb{C}^{2} \times T^{*} S, \\
z & \longmapsto\left(\left(-t_{0}, 2 \sqrt{t_{0}} z\right),\left(q_{0}, 0\right)\right),
\end{aligned}
$$

where $0 \leq t_{0}<C$ is a real parameter and $q_{0} \in S$. The maps $u_{\left(t_{0}, q_{0}\right)}$ are $(i \times j)$ holomorphic, where $j$ denotes the natural almost complex structure on $T^{*} S$ induced by the Levi-Civita connection of a Riemannian metric on $S$. Moreover, they have boundary on the set corresponding to $\mathcal{P} S(S)$.

We denote by $J$ the almost complex structure on $V$ obtained by pulling back the almost complex structure $i \times j$ from $\mathbb{C}^{2} \times T^{*} S$. Then we can pull back the Bishop family to holomorphic maps (denoted by the same symbols)

$$
u_{t_{0}, q_{0}}: \mathbb{D}^{2} \longrightarrow V \subset(-\varepsilon, 0] \times M .
$$


2.3. Uniqueness results for holomorphic disks. We extend the almost complex structure $J$ from the set $V$ to a compatible almost complex structure on $(W:=$ $\left.(-\infty, 0] \times M, d\left(e^{s} \lambda\right)\right)$. We introduce the notation

$$
\widehat{\mathcal{P S}}(S)=\mathcal{P S}(S) \backslash(\partial \mathcal{P S}(S) \cup S)
$$

and remark that $\widehat{\mathcal{P S}}(S)$ is totally real with respect to $J$. The following proposition is taken from [13, Proposition 7].

Proposition 2.1. Let $u:\left(\mathbb{D}^{2}, \partial \mathbb{D}^{2}\right) \rightarrow(W, \widehat{\mathcal{P S}}(S))$ be a J-holomorphic disk which is simple. Moreover, we assume that $u\left(S^{1}\right) \subset \mathcal{P S}(S)$ bounds a disk in $\mathcal{P S}(S)$ and

$$
\operatorname{image}(u) \cap V \neq \varnothing .
$$

Then, up to an element in $\operatorname{Aut}\left(\mathbb{D}^{2}\right)$, we have

$$
u=u_{t_{0}, q_{0}},
$$

that is, after reparametrization, the holomorphic disk $u$ is a member of the Bishop family.

\section{Proof of the theorem}

By assumption there exists a contact form $\lambda_{\text {PS }}$ on $M$ containing a Plastikstufe. Let $\lambda$ be another contact form inducing the same contact structure.

We assume by contradiction that there exists no contractible closed Reeb orbit for $\lambda$.

3.1. The set-up. We choose a function $f: M \rightarrow \mathbb{R}$ such that $\lambda=f \lambda_{\mathrm{PS}}$. Since multiplying $\lambda$ with a non-zero constant does not change its Reeb orbits (up to reparametrization) we may assume without loss of generality that the function $f$ takes only values in $(0,1)$. Then we can choose a smooth family of functions $f_{s}: M \rightarrow(0, \infty)$ for $s \in[-1,-\varepsilon]$ satisfying

$$
f_{s}=\left\{\begin{array}{ll}
1 & \text { near } s=-\varepsilon, \\
f & \text { near } s=-1,
\end{array} \quad \text { and moreover } \quad \frac{\partial f_{s}}{\partial s} \geq 0 .\right.
$$

This gives rise to a smooth family $\lambda_{s}=f_{s} \lambda_{\mathrm{PS}}$ of contact forms which we extend by $\lambda_{\text {PS }}$ for $s \geq-\varepsilon$ and by $\lambda$ for $s \leq-1$. On $W=(-\infty, 0] \times M$ we choose an exact symplectic form $\Omega$ which satisfies

$$
\Omega= \begin{cases}d\left(e^{s} \lambda_{\mathrm{PS}}\right) & \text { on }[-\varepsilon, 0] \times M, \\ d\left(e^{s} \lambda\right) & \text { on }(-\infty,-1] \times M\end{cases}
$$


This is possible due to our choice of the family $f_{s}$. This has been used in the literature many times, see for instance [7]. We modify the almost complex structure $J$ from above to a compatible almost complex structure $J$ on $(W, \Omega)$ by requiring that on $(-\infty,-2] \times M$ the almost complex structure is adapted to the negative part of the symplectization of $\lambda$, in the sense of [1]. On $V$ it remains as defined in the previous section. In particular, $(W, \Omega)$ still contains the Bishop family $u_{t_{0}, q_{0}}$. We denote the relative homotopy class given by the Bishop disks by $a \in \pi_{2}(W, \widehat{\mathcal{P} S}(S))$ and set

$$
\begin{aligned}
& \mathcal{M}(J):=\left\{u:\left(\mathbb{D}^{2}, \partial \mathbb{D}^{2}\right) \longrightarrow(W, \widehat{\mathcal{P} S}(S)) \mid \bar{\partial}_{J} u=0,[u]=a, \operatorname{lk}(u, S)=1\right\} \\
& \hat{\mathcal{M}}(J):=\mathcal{M}(J) / \operatorname{Aut}\left(\mathbb{D}^{2}\right),
\end{aligned}
$$

where $\operatorname{lk}(u, S)$ is the linking number of $u\left(S^{1}\right)$ in $\mathcal{P S}(S)$ with the set $S$. This is defined as follows. By definition $\widehat{\mathcal{P S}}(S)$ is foliated by an $S^{1}$-family of Legendrian submanifolds, thus there exists a natural map $\theta: \widehat{\mathcal{P S}}(S) \rightarrow S^{1}$. We set $\operatorname{lk}(u, S):=$ $\operatorname{deg}\left(\left.\theta \circ u\right|_{S^{1}}\right)$.

3.2. The proof. We need the following three facts established in Propositions $8-10$ in [13].

(1) The Maslov index of $a$ equals $\mu_{\text {Maslov }}(a)=2$,

(2) the almost complex structure $J$ is regular at members of the Bishop family,

(3) the energy of all elements in $\mathcal{M}(J)$ is uniformly bounded.

The totally real submanifold $\widehat{\mathcal{P} S}(S)$ is non-compact. Since $\partial \mathcal{P S}(S)$ is a closed leaf of the characteristic foliation the maximum principle implies that no holomorphic maps intersect $\partial \mathcal{P S}(S)$ at an interior point. According to Proposition 2.1 near $S$ the only holomorphic disks are members of the Bishop family. Therefore, the noncompactness of $\widehat{\mathcal{P S}}(S)$ poses no problem. Moreover, due to the energy bounds and the specific structure of the almost complex structure $J$ on the end of $W$ we can apply the ideas of the SFT-compactness theorem [1]. Since we assumed that there exists no contractible closed Reeb orbits bubbling-off cannot occur in the interior. Therefore, the only non-compactness of the moduli space $\widehat{\mathcal{M}}(J)$ comes from bubbling-off of holomorphic disks having boundary on $\widehat{\mathcal{P S}}(S)$. The next proposition is taken from [13, Proposition 11] and shows that there exists no bubbling-off of holomorphic disks.

Proposition 3.1. Given a sequence $\left(u_{n}\right) \subset \widehat{\mathcal{M}}(J)$ there exists a subsequence either converging to an element in $\widehat{\mathcal{M}}(J)$ or to a point in $S$.

The latter case occurs if a family of Bishop disks shrinks to a point in $S$. We remark that in the former case the limit is simple. 
Proposition 3.2. For a compatible almost complex structure $J$, which is generic on the subset $((-2,0] \times M) \backslash V$ of $(W, \Omega)$, the moduli space $\mathcal{M}(J)$ is a smooth manifold of dimension

$$
\operatorname{dim} \mathcal{M}(J)=n+2 .
$$

Proof. We pick $u \in \mathcal{M}(J)$. In case that image $(u) \cap V \neq \varnothing$ we conclude from Proposition 2.1 that $u$ is a member of the Bishop family. In particular, image $(u) \subset V$. Moreover, $J$ is already regular for members in the Bishop family.

If image $(u) \cap V=\emptyset$ then $u$ has to pass through the region $((-2,0] \times M) \backslash V$. Since all the disks are simple a generic $J$ will be regular, see for example [2]. The dimension formula follows from the fact that $\mu_{\text {Maslov }}(a)=2$ and $\operatorname{dim} \widehat{\mathcal{P S}}(S)=n$.

We consider the evaluation map

$$
\begin{aligned}
\mathrm{ev}: \hat{\mathcal{M}}(J)_{S^{1}}:=\mathcal{M}(J) \times_{\mathrm{Aut}\left(\mathbb{D}^{2}\right)} S^{1} & \longrightarrow \widehat{\mathcal{P} S}(S) \subset M, \\
{[u, t] } & \longmapsto u\left(e^{2 \pi i t}\right),
\end{aligned}
$$

defined on the smooth manifolds $\widehat{\mathcal{M}}(J)_{S^{1}}$ of dimension $\operatorname{dim} \widehat{\mathcal{M}}(J)_{S^{1}}=n$. The following proposition is proved analogously to the transversality result above.

Proposition 3.3. For a generic $J$ as in the previous proposition the evaluation map is smooth.

To derive the contradiction to the assumption that $\lambda$ has no closed Reeb orbits we make the following

Definition 3.4. For a point $p=\iota(z, s) \in \iota\left(\mathbb{D}^{2} \times S\right)=\mathcal{P S}(S)$ we define the distance of $p$ to $S$ by $d(p, S)=|z|$ and set for $\delta>0$ sufficiently small

$$
\widehat{\mathcal{M}}(J)_{S^{1}}^{\delta}:=\left\{[u, t] \in \widehat{\mathcal{M}}(J)_{S^{1}} \mid d(\operatorname{ev}([u, t]), S) \geq \delta\right\} .
$$

Then we have by construction of the Bishop family

$$
\operatorname{ev}\left(\partial \hat{\mathcal{M}}(J)_{S^{1}}^{\delta}\right)=\iota\left(S_{\delta}^{1} \times S\right),
$$

where $S_{\delta}^{1}=\left\{z \in \mathbb{D}^{2}|| z \mid=\delta\right\}$. We conclude that

$$
\left[\operatorname{ev}\left(\partial \widehat{\mathcal{M}}(J)_{S^{1}}^{\delta}\right)\right] \in \mathrm{H}_{n-1}(\widehat{\mathcal{P S}}(S), \mathbb{Z} / 2)
$$

is the generator. On the other hand the set $\operatorname{ev}\left(\partial \hat{\mathcal{M}}(J)_{S^{1}}^{\delta}\right)$ is clearly the boundary of the compact, see Proposition 3.1, manifold $\operatorname{ev}\left(\widehat{\mathcal{M}}(J)_{S^{1}}^{\delta}\right)$. This implies, that

$$
\left[\operatorname{ev}\left(\partial \widehat{\mathcal{M}}(J)_{S^{1}}^{\delta}\right)\right]=0 \in \mathrm{H}_{n-1}(\widehat{\mathcal{P} S}(S), \mathbb{Z} / 2) .
$$

This contradictions concludes the proof of the theorem. 


\section{References}

[1] F. Bourgeois, Y. Eliashberg, H. Hofer, K. Wysocki, and E. Zehnder, Compactness results in symplectic field theory. Geom. Topol. 7 (2003), 799-888. Zbl 1131.53312 MR 2026549

[2] D. L. Dragnev, Fredholm theory and transversality for noncompact pseudoholomorphic maps in symplectizations. Comm. Pure Appl. Math. 57 (2004), 726-763. Zbl 1063.53086 MR 2038115

[3] M. Gromov, Pseudoholomorphic curves in symplectic manifolds. Invent. Math. 82 (1985), 307-347. Zbl 0592.53025 MR 0809718

[4] H. Hofer, Pseudoholomorphic curves in symplectizations with applications to the Weinstein conjecture in dimension three. Invent. Math. 114 (1993), 515-563. Zbl 0797.58023 MR 1244912

[5] - A general Fredholm theory and Applications. In Proceedings of the CDM (Harvard), 2004.

[6] H. Hofer, K. Wysocki, and E. Zehnder, Pseudoholomorphic curves and dynamics in three dimensions. In Handbook of dynamical systems, Vol. 1A, North-Holland, Amsterdam 2002, 1129-1188. Zbl 1052.53063 MR 1928532

[7] — Finite energy foliations of tight three-spheres and Hamiltonian dynamics. Ann. of Math. (2) 157 (2003), 125-255. Zbl 01933157 MR 1954266

[8] — A General Fredholm Theory I: A Splicing-Based Differential Geometry. J. Eur. Math. Soc. (JEMS) 9 (2007), 841-876. Zbl 05255318 MR 2341834

[9] —- A General Fredholm Theory II: Implicit Function Theorems. Geom. Funct. Anal., to appear; arXiv:0705.1310v2.

[10] —, Applications of Polyfold Theory I: Gromov-Witten Theory. Article in preparation.

[11] M. Hutchings, An index inequality for embedded pseudoholomorphic curves in symplectizations. J. Eur. Math. Soc. (JEMS) 4 (2002), 313-361. Zbl 1017.58005 MR 1941088

[12] P. B. Kronheimer and T. S. Mrowka, Floer homology for Seiberg-Witten monopoles. Cambrigde University Press, to appear.

[13] K. Niederkrüger, The plastikstufe - a generalization of the overtwisted disk to higher dimensions. Algebr. Geom. Topol. 6 (2006), 2473-2508. Zbl 1129.53056 MR 2286033

[14] K. Niederkrüger and O. van Koert, Every contact manifold can be given a non-fillable contact structure. Internat. Math. Res. Not. (2007), article ID rnm115. Zbl 1133.53053 MR 2380008

[15] F. Presas, A class of non-fillable contact structures. Geom. Topol. 11 (2007), 2203-2225. Zbl 1132.57023 MR 2372846

[16] P. H. Rabinowitz, Periodic solutions of a Hamiltonian system on a prescribed energy surface. J. Differential Equations 33 (1979), 336-352. Zbl 0424.34043 MR 0543703

[17] C. H. Taubes, The Seiberg-Witten equations and the Weinstein conjecture. Geom. Topol. 11 (2007), 2117-2202. Zbl 1135.57015 MR 2350473

[18] — The Seiberg-Witten equations and the Weinstein conjecture II: More closed integral curves of the Reeb vector field. Preprint 2007; arXiv:math/0702366v2. 
[19] C. Viterbo, A proof of Weinstein's conjecture in $\mathbf{R}^{2 n}$. Ann. Inst. H. Poincaré Anal. Non Linéaire 4 (1987), 337-356. Zbl 0631.58013 MR 0917741

[20] A. Weinstein, Periodic orbits for convex Hamiltonian systems. Ann. of Math. (2) 108 (1978), 507-518. Zbl 0403.58001 MR 0512430

[21] _ - On the hypotheses of Rabinowitz' periodic orbit theorems. J. Differential Equations 33 (1979), 353-358. Zbl 0388.58020 MR 0543704

Received June 9, 2007

Peter Albers, Courant Institute, New York University, 251 Mercer Street, NY 10012, U.S.A.

E-mail: albers@cims.nyu.edu

Helmut Hofer, Courant Institute, New York University, 251 Mercer Street, NY 10012, U.S.A.

E-mail: hofer@cims.nyu.edu 